\title{
Erratum to: Growth and Nutrient Composition of Platycodon Grown in Media Amended with Pellets Processed with Poultry Feathers
}

\author{
In Kyung Kim ${ }^{1}$, Mark S. Roh ${ }^{2}$, Yong Seung Roh ${ }^{1}$, and Yong Kwon Yoo ${ }^{1,2^{*}}$ \\ ${ }^{1}$ Mokpo National University, Institute of Natural Resources, Muan 534-729, Korea \\ ${ }^{2}$ Mokpo National University, Department of Horticultural Science, Muan 534-729, Korea \\ *Corresponding author: yooyong@mokpo.ac.kr
}

(C) Korean Society for Horticultural Science and Springer 2017

Erratum to: Hortic. Environ. Biotechnol. 58(3):218-230. 2017.

DOI 10.1007/s13580-017-0073-9

The original version of this article contained errors. The correct version should be written as shown in the following table.

\begin{tabular}{|c|c|c|c|c|}
\hline Page & Column & Line & Before correction & After correction \\
\hline p.218 & & line 3 & mixing them & mixing \\
\hline p.218 & & line 5 & plant culture & culture \\
\hline p.219 & Left & line 11 & is processed & processed \\
\hline p.219 & Left & line 13 & mediumfor & medium for \\
\hline p.219 & Left & line 16 & Corylopsiscoreana & Corylopsis coreana \\
\hline p.220 & Table 1 & & $\begin{array}{l}\text { Odor } \\
\text { neutralize } \\
r(\%)\end{array}$ & $\begin{array}{l}\text { Odor } \\
\text { neutralizer } \\
(\%)\end{array}$ \\
\hline p.220 & Right & line 30 & $p=$ & $p<$ \\
\hline p.221 & Left & line 4 & werethe & were the \\
\hline p.222 & Table 3 & line 1 & micro-nutrients inleachate & micro-nutrients in leachate \\
\hline p.222 & & line 2 & $p=$ & $p<$ \\
\hline p.222 & Right & line 9 & inplants & in plants \\
\hline p.223 & Table 4 & & Level ofsignificance & Level of significance \\
\hline p.223 & & line 3 & $p=$ & $p<$ \\
\hline p.223 & Left & line 2 & inplants & in plants \\
\hline p.224 & & line 2 & $p=$ & $p<$ \\
\hline p.224 & & line 3 & range ssuggested & ranges suggested \\
\hline p.224 & Right & line 13 & foreach & for each \\
\hline p.225 & & line 2 & $p=$ & $p<$ \\
\hline p.225 & Right & line 10 & amendedwith & amended with \\
\hline p.225 & Right & line 12 & Osmocote(Baker) & Osmocote (Baker) \\
\hline p.226 & Left & line 2 & Investigate the a & Investigate the \\
\hline p.226 & Table 7 & line 1 & amedium & a medium \\
\hline p.226 & & line 4 & $p=$ & $p<$ \\
\hline p.226 & & line 7 & vapted & ${ }^{v}$ Adapted \\
\hline p.226 & Right & line 2 & Passiflora & Passiflora \\
\hline p.226 & Right & line 4 & massin & mass in \\
\hline p.227 & Right & line 7 & branchesand & branches and \\
\hline p.228 & Left & line 7 & Glycine $\max$ & Glycine max \\
\hline p.228 & Left & line 9 & wasnot & was not \\
\hline p.228 & Left & line 12 & thatprevents & that prevents \\
\hline p.228 & Right & line 41 & apotted plant, a & a potted plant, an \\
\hline p.228 & Right & line 42 & duringthe & during the \\
\hline p.229 & Right & line 2-3 & suggested as such a fertilizer. & suggested. \\
\hline p.229 & Right & line 10 & aregreatly & are greatly \\
\hline p.229 & Right & line 23 & Sta Man Manual 23 & Sta Manual 23 \\
\hline p.230 & Left & line 10 & (1966)Silica & (1966) Silica \\
\hline p.230 & Right & line 18 & 2015.http & 2015. http \\
\hline p.230 & Right & line 18 & Proceedings & Proc \\
\hline p.230 & Right & line 27 & HortScience25 & HortScience 25 \\
\hline
\end{tabular}

The online version of the original article can be found under doi:10.1007/s13580-017-0073-9 